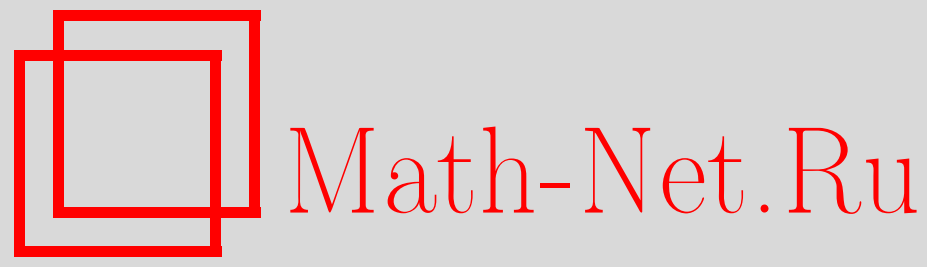

О. Е. Тихонов, О сходимости функций нормальных операторов в сильной операторной топологии, Функи. анализ и его прил., 2007, том 41, выпуск $3,93-95$

DOI: https://doi.org/10.4213/faa2871

Использование Общероссийского математического портала MathNet.Ru подразумевает, что вы прочитали и согласны с пользовательским соглашением http://www . mathnet.ru/rus/agreement

Параметры загрузки:

IP : 35.173 .219 .12

26 апреля 2023 г., 15:33:08

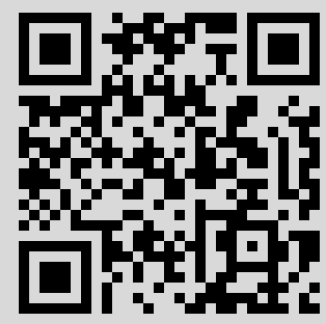


[11] Б. М. Левитан, В. В. Жиков, Почти периодические функиии и дифференциальные уравнения, Изд-во МГУ, М., 1978. [12] L. H. Loomis, Ann. Math. (2), 72 (1960), 362-368. [13] Ю. И. Любич, Введение в теорию банаховых представлений групп, Высшая школа, Харьков, 1985. [14] H. S. Mustafayev, Bull. Polish. Acad. Sci. Math., 52:4 (2004), 395-403. [15] H. Reiter, Classical Harmonic Analysis and Locally Compact Groups, Clarendon Press, Oxford, 1968. [16] C. Rickart, General Theory of Banach Algebras, Van Nostrand, Princeton, 1960.

Факультет естественных и гуманитарных наук Университета Йузунджу Йыл, Турция

Поступило в редакцию e-mail: hsmustafayev@yahoo.com

28 июня 2005 г.

УДК 517.983.243, 517.984.4

\title{
О сходимости функций нормальных операторов в сильной операторной топологии*
}

\author{
(c) 2007. О. Е. Тихонов
}

Всюду ниже $\mathscr{N}(H)$ обозначает множество всех ограниченных нормальных операторов в гильбертовом пространстве $H$, а $\operatorname{Sp}(A)$ - спектр оператора $A$. Для числовой функции $f$ соответствующая ей по спектральной теореме операторная функция обозначается той же буквой. Символ $\stackrel{s}{\rightarrow}$ используется для обозначения сходимости в сильной операторной топологии (далее - в $s$-топологии).

В работе Дэвиса [1] доказана следующая

Теорема. Пусть $A, A_{n} \in \mathscr{N}(H)$, и пусть $A_{n} \stackrel{s}{\rightarrow}$ A. Пусть $f-$ ограниченная борелевская функиия, определенная на $\mathbb{C}, u \Gamma-$ замкнутое подмножество в $\operatorname{Sp}(A)$, спектральный проектор которого относительно А равен нулю. Пусть $f$ непрерьвна в каждой точке из $\mathrm{Sp}(A) \backslash \Gamma$. Тогда $f\left(A_{n}\right) \stackrel{s}{\rightarrow} f(A)$.

Цель настоящей заметки - обобщить эту теорему таким образом, чтобы получить уточнение теоремы Кэдисона [2] о сильной непрерывности операторных функций на подмножествах нормальных операторов. При доказательстве применяются результаты и конструкции работ [1], [2]. Существенно новый момент - использование следующей топологической леммы.

Лемма 1. Пусть $(X, d)$ - такое метрическое пространство, что все его

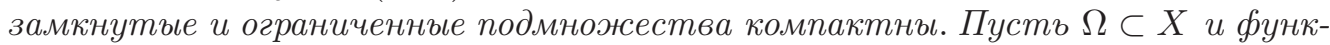
иия $f: \Omega \rightarrow[a, b]$ непрерьвна на $\Omega$. Тогда существует функиия $g: X \rightarrow[a, b]$, совпадающая с $f$ на $\Omega$, непрерьвная во всех точках из $\Omega$ и полунепрерывная сверху на $X$.

Доказательство. Для произвольной точки $x^{\prime}$ из замыкания $\bar{\Omega}$ множества $\Omega$ положим $g_{1}\left(x^{\prime}\right)=\lim \sup _{x \in \Omega, x \rightarrow x^{\prime}} f(x)$. Через $\operatorname{dist}(x, \Delta)$ обозначим расстояние между точкой $x \in X$ и множеством $\Delta \subset X$. Для произвольного $x \in X$ рассмотрим непустое компактное множество $\Omega_{x}=\left\{x^{\prime} \in \bar{\Omega} \mid d\left(x, x^{\prime}\right)=\operatorname{dist}(x, \bar{\Omega})\right\}$ и положим $g(x)=\sup \left\{g_{1}\left(x^{\prime}\right) \mid x^{\prime} \in \Omega_{x}\right\}$. Стандартным образом проверяется, что функция $g$ удовлетворяет всем требуемым условиям.

*Работа поддержана РФФИ, проект 05-01-00799. 
Лемма 2. Пусть $\Delta$ - замкнутое подмножество метрического пространства $(X, d)$. Пусть функиия $f: X \rightarrow \mathbb{R}$ ограничена, равна 0 на $\Delta$ и непрерывна в каждой точке из $\Delta$. Тогда существует ограниченная непрерывная функиия $h$ на $X$, mакая, что $h=0$ на $\Delta u|f| \leqslant h$ на $X$.

Доказательство. Будем считать, что $|f(x)| \leqslant 1$ для всех $x \in X$. Следуя [1], рассмотрим множества $X_{n}=\left\{x \in X|| f(x) \mid \geqslant 2^{-n}\right\}$ и положим $h(x)=$ $\sum_{n=0}^{\infty} 2^{-n} \operatorname{dist}(x, \Delta) /\left(\operatorname{dist}(x, \Delta)+\operatorname{dist}\left(x, \bar{X}_{n}\right)\right)$. Тогда функция $h$ обладает всеми требуемыми свойствами.

Далее, для $\Omega \subset \mathbb{C}$ положим $\mathscr{N}(H)_{\Omega}=\{A \in \mathscr{N}(H) \mid \operatorname{Sp}(A) \subset \Omega\}$. При доказательстве основного результата мы будем использовать следующий ослабленный вариант теоремы 4.2 из [2].

Предложение 3. Пусть $f: \mathbb{C} \rightarrow \mathbb{C}$ - непрерывная функиия, такая, что $\sup \{|f(z)| /(1+|z|) \mid z \in \mathbb{C}\}<+\infty$. Тогда операторная функиия $f: \mathscr{N}(H) \rightarrow$ $\mathscr{N}(H)$ непрерывна на $\mathscr{N}(H)$ в s-топологии.

Теорема 4. Пусть $\Omega \subset \mathbb{C}, A \in \mathscr{N}(H)_{\Omega}$ и $\Gamma$ - замкнутое подмножество в $\operatorname{Sp}(A)$, спектральный проектор которого относительно А равен нулю. Пусть функиия $f: \Omega \rightarrow \mathbb{C}$ такова, что ее ограничение на любое ограниченное замкнутое в $\mathbb{C}$ подмножество множества $\Omega$ измеримо по Борелю, $\sup \{|f(z)| /(1+|z|) \mid z \in \Omega\}<+\infty$ и $f$ непрерывна в каждой точке из $\operatorname{Sp}(A) \backslash \Gamma$. Тогда, если сеть $\left\{A_{\alpha}\right\}$ операторов из $\mathscr{N}(H)_{\Omega}$ сходиться $\kappa$ А в s-топологии, mo $f\left(A_{\alpha}\right) \stackrel{s}{\rightarrow} f(A)$.

Доказательство. Теорему достаточно доказать для вещественнозначной функции $f$, что мы и проделаем ниже в три этапа.

1. Предположим, что $f$ ограничена на $\Omega$, скажем $|f| \leqslant 1$, и непрерывна во всех точках из $\operatorname{Sp}(A)$. По теореме Титце-Урысона существует непрерывная функция $g: \mathbb{C} \rightarrow[-1,1]$, совпадающая с $f$ на $\operatorname{Sp}(A)$. По предложению 3 $g\left(A_{\alpha}\right) \stackrel{s}{\rightarrow} g(A)=f(A)$. По лемме 2 существует ограниченная непрерывная функция $h$ на $\Omega$, такая, что $h=0$ на $\operatorname{Sp}(A)$ и $|f-g| \leqslant h$ на $\Omega$.

1а. Теперь предположим дополнительно, что $\Omega=\mathbb{C}$. Тогда $h\left(A_{\alpha}\right) \stackrel{s}{\rightarrow} h(A)=0$ по предложению 3 . Так как $0 \leqslant(f-g+h)\left(A_{\alpha}\right) \leqslant 2 h\left(A_{\alpha}\right)$, то $(f-g+h)\left(A_{\alpha}\right) \stackrel{s}{\rightarrow} 0$. Следовательно, $f\left(A_{\alpha}\right)=(f-g+h)\left(A_{\alpha}\right)+g\left(A_{\alpha}\right)-h\left(A_{\alpha}\right) \stackrel{s}{\rightarrow} f(A)$.

$1 \mathrm{~b}$. Если $\Omega \neq \mathbb{C}$, то, согласно лемме 1 , существует ограниченная функция $k: \mathbb{C} \rightarrow \mathbb{R}$, которая продолжает $h$, непрерывна во всех точках из $\Omega$ и полунепрерывна сверху на $\mathbb{C}$, а следовательно, измерима по Борелю. Воспользовавшись случаем 1a, получаем, что $h\left(A_{\alpha}\right)=k\left(A_{\alpha}\right) \stackrel{s}{\rightarrow} k(A)=h(A)=0$, и остается дословно повторить два последних предложения предыдущего абзаца.

2. Переход от ограниченных функций, непрерывных во всех точках из $\operatorname{Sp}(A)$, к ограниченным функциям, которые на $\operatorname{Sp}(A)$ могут иметь точки разрыва, осуществляется точно так же, как в [1].

3. В общем случае положим $g(z)=f(z) /(1+|z|)$. Тогда $g\left(A_{\alpha}\right) \stackrel{s}{\rightarrow} g(A)$ по предыдущему и $1+\left|A_{\alpha}\right| \stackrel{s}{\rightarrow} 1+|A|$ по предложению 3. Отсюда выводим, что $f\left(A_{\alpha}\right)=g\left(A_{\alpha}\right)\left(1+\left|A_{\alpha}\right|\right) \stackrel{s}{\rightarrow} g(A)(1+|A|)=f(A)$.

Следствие 5. Пусть $\Omega \subset \mathbb{C}$, функиия $f: \Omega \rightarrow \mathbb{C}$ непрерывна на $\Omega$ u $\sup \{|f(z)| /(1+|z|) \mid z \in \Omega\}<+\infty$. Тогда операторная функиия $f: \mathscr{N}(H)_{\Omega} \rightarrow$ $\mathscr{N}(H)$ непрерывна на $\mathscr{N}(H)_{\Omega}$ в s-топологии. 
Следствие 5 уточняет теорему 4.2 из [2], в которой на множество $\Omega$ налагалось ограничение $\overline{(\bar{\Omega} \backslash \Omega}) \cap \Omega=\varnothing$. С этим или более сильным ограничением упомянутая теорема приводится в ряде монографий по теории операторных алгебр (например, [3, теорема II.4.7], [4, А.2]). Заметим еще, что для самосопряженных операторов результат, аналогичный следствию 5 , был приведен в [5, теорема 2.7].

Автор выражает признательность А. Н. Шерстневу, привлекшему в свое время его внимание к задачам непрерывности операторных функций.

\section{Список литературы}

[1] E. B. Davies, J. London Math. Soc. (2), 4 (1972), 435-436. [2] R. V. Kadison, Pacific J. Math., 26 (1968), 121-129. [3] M. Takesaki, Theory of operator algebras I, Springer-Verlag, Berlin-Heidelberg-New York, 1979. [4] S. Strătilă, Modular theory in operator algebras, Editura Academiei, Bucuresti; Abacus Press, Tunbridge Wells, Kent, 1981. [5] О. Е. Тихонов, Изв. ВУЗов, матем., 1987, № 1, 77-79.

НИИ математики и механики им. Н. Г. Чеботарева Казанского государственного университета e-mail: Oleg.Tikhonov@ksu.ru
Поступило в редакцию 3 февраля 2006 г. 